\title{
Circadian blood pressure changes and cardiovascular risk in elderly-treated hypertensive patients
}

\author{
Sante D Pierdomenico ${ }^{1,2}$, Anna M Pierdomenico ${ }^{2,3}$, Francesca Coccina ${ }^{3}$, Domenico Lapenna ${ }^{2,3}$ \\ and Ettore Porreca ${ }^{1,2}$
}

The independent prognostic significance of circadian blood pressure (BP) changes is unclear. We investigated the association between circadian BP changes and cardiovascular risk among elderly-treated hypertensive patients. The occurrence of a composite end point (that is, stroke, coronary events, heart failure and peripheral revascularization) was evaluated among 1191 elderly-treated hypertensive patients (age range 60-90 years). According to the nighttime change and the morning surge (MS) of systolic BP, subjects were divided into groups of dippers with a normal or high MS (DNMS and DHMS, respectively), non-dippers (ND), reverse dippers (RD) and extreme dippers with a normal or high MS (EDNMS and EDHMS, respectively). During the follow-up $(9.1 \pm 4.9$ years, range $0.4-20$ years), 392 events occurred. The event rate was 3.63 per 100 patient-years. After adjustment for various covariates, including 24-h BP, the DHMS (hazard ratio (HR) $1.49,95 \%$ confidence interval $(\mathrm{Cl})$ 1.02-2.16, $P=0.04$ ), ND (HR 1.71, 95\% Cl 1.28-2.27, $P=0.0001$ ), RD (HR 2.05, 95\% Cl 1.44-2.93, $P=0.0001$ ) and EDHMS (HR 3.40, 95\% Cl 1.96-5.90, $P=0.001$ ) were at higher cardiovascular risk than the DNMS. The population attributable risk was $0.6,7.1,7.3$ and $1.4 \%$ for the DHMS, ND, RD and EDHMS, respectively. In elderly-treated hypertensive patients, circadian BP changes were independently associated with increased cardiovascular risk. At the patient level, the highest risk was observed among the EDHMS, followed by the RD, ND and DHMS. At the population level, the highest risk was observed among the RD, followed by the ND, EDHMS and DHMS.

Hypertension Research (2016) 39, 805-811; doi:10.1038/hr.2016.74; published online 23 June 2016

Keywords: ambulatory blood pressure; cardiovascular events; dippers; morning surge; non-dippers

\section{INTRODUCTION}

Ambulatory blood pressure (BP) monitoring is increasingly used in clinical practice. ${ }^{1}$

Ambulatory BP is largely superior to clinic BP in predicting the prognosis of both untreated and treated hypertensive patients at baseline. $^{1-5}$ Ambulatory BP monitoring has also revealed that the circadian BP profile is not uniform among hypertensive subjects. Indeed, for nighttime BP behavior, the following circadian BP patterns have been described: (1) reverse dipper (BP fall $<0 \%$ ), (2) non-dipper (BP fall $\geqslant 0 \%$ and $<10 \%$ ), (3) dipper (BP fall $\geqslant 10 \%$ and $<20 \%$ ) and (4) extreme dipper (BP fall $\geqslant 20 \%$ ). Various studies have evaluated cardiovascular outcomes according to circadian BP patterns among both hypertensive patients ${ }^{6-19}$ and general populations, ${ }^{20-22}$ but the findings are not consistent, and all circadian BP patterns were not analyzed in each study. Moreover, ambulatory BP monitoring has also presented the opportunity to evaluate the morning surge (MS) of BP, that is, the difference between BP after and before awakening, which has been implicated in the occurrence of cardiovascular events, given their reported peak incidence in the morning. ${ }^{23,24}$ Some investigators have evaluated the association between a high MS of BP and future cardiovascular complications among both hypertensive patients ${ }^{8,19,25-31}$ and general populations, ${ }^{22,32-34}$ but the results are conflicting.

Notably, non-dippers show a lower MS of BP, whereas dippers show a higher MS of BP. Thus, it is problematic to reconcile the adverse prognostic significance of non-dipping with the statement that a high MS of BP is also a predictor of poor outcome when hypertensive patients are analyzed as a group. We suggest that dippers and non-dippers should be analyzed separately with group-specific cutoff points, and we have recently reported that a high MS of BP predicts stroke, ${ }^{28}$ coronary events ${ }^{30}$ and heart failure with reduced ejection fraction $(\mathrm{EF})^{31}$ in dippers but not in non-dippers or hypertensive patients as a group.

Altogether, previous studies have reported conflicting results; some of them have not analyzed all circadian BP patterns, the majority of them have not analyzed the MS of BP according to dipping status, and no study has specifically evaluated an elderly-treated hypertensive population. The aim of this study was to investigate the relationship between incidence of cardiovascular events and circadian BP pattern (that is, reverse dipper, non-dipper, dipper with a normal or high MS

${ }^{1}$ Dipartimento di Scienze Mediche, Orali e Biotecnologiche, Università “Gabriele d’Annunzio", Chieti-Pescara, Italy; ${ }^{2}$ Centro di Ricerca Clinica, Fondazione Università "Gabriele d'Annunzio", Chieti-Pescara, Italy and "Dipartimento di Medicina e Scienze dell'Invecchiamento, Università "Gabriele d'Annunzio", Chieti-Pescara, Italy Correspondence: Professor SD Pierdomenico, Patologia Medica, Policlinico "Santissima Annunziata", Via dei Vestini, Chieti Scalo (Chieti) 66013, Italy. E-mail: pierdomenico@unich.it or santedonato.pierdomenico@fastwebnet.it

Received 28 February 2016; revised 17 April 2016; accepted 11 May 2016; published online 23 June 2016 
and extreme dipper with a normal or high MS) among elderly-treated hypertensive patients.

\section{METHODS}

\section{Subjects}

Since 1992, we have built two prospective databases of our initially untreated or initially treated hypertensive patients for the purpose of evaluating the prognostic value of ambulatory BP parameters and other risk markers. The present study was carried out with the database of initially treated subjects. We studied 1191 sequentially treated hypertensive patients aged $\geqslant 60$ years (range 60-90 years) who were prospectively recruited from December 1992 to December 2012 and referred to our hospital outpatient clinic for evaluation of BP control. Sixty-two patients were lost during follow-up. Subjects with secondary hypertension were excluded. All the patients underwent clinical evaluations, electrocardiograms, routine laboratory tests, echocardiographic examinations and non-invasive ambulatory BP monitoring. The study population came from the same geographical area (Chieti and Pescara, Abruzzo, Italy). The study was in accordance with the Second Declaration of Helsinki and was approved by the institutional review committee. The subjects gave informed consent.

\section{Office BP measurements}

Clinic systolic and diastolic BP recordings were collected by a physician who used a mercury sphygmomanometer and appropriate-sized cuffs. Phase V was used to determine the diastolic BP. The measurements were performed in triplicate, 2 min apart, and the mean value was used as the BP for the visit.

\section{Ambulatory BP monitoring}

Ambulatory BP monitoring was conducted with a portable, non-invasive recorder (SpaceLabs 90207, Redmond, WA, USA) on a day of typical activity within 1 week of the clinic BP measurement. Each time that a reading was taken, the subjects were instructed to remain motionless and to record their activity on a diary sheet. The technical aspects have been previously reported. ${ }^{35}$ Ambulatory BP readings were obtained at 15 -min intervals from $0600 \mathrm{~h}$ to midnight and at 30-min intervals from midnight to $0600 \mathrm{~h}$. The following ambulatory BP parameters were evaluated: daytime (awake period), nighttime (asleep period) and 24-h systolic and diastolic BP; weighted standard deviation (s.d.) of 24-h BP as an index of BP variability, ${ }^{36}$ according to the formula $(($ daytime s.d. $\times$ awake time in hours $)+($ nighttime s.d. $\times$ asleep time in hours $)) /$ 24; the extent of BP reduction from day to night (those with BP fall $<0 \%$ were defined as reverse dippers, those with $\mathrm{BP}$ fall $\geqslant 0$ and $<10 \%$ as non-dippers, those with BP fall $\geqslant 10$ and $<20 \%$ as dippers and those with BP fall $\geqslant 20 \%$ as extreme dippers); and the pre-awakening MS of BP defined as the difference between the mean BP during the $2 \mathrm{~h}$ after waking and the mean BP during the $2 \mathrm{~h}$ before waking, evaluated separately among the dippers and non-dippers. As previously reported ${ }^{28,30,31}$ in this population, we divided all the non-dippers (reverse dippers and non-dippers) and all the dippers (dippers and extreme dippers) according to group-specific tertiles of the MS of systolic $\mathrm{BP}$; the dippers (dippers and extreme dippers) with a MS in the upper tertile $(>23 \mathrm{~mm} \mathrm{Hg}$ ) were defined as having a high MS. The recordings were automatically edited (that is, excluded) if the systolic BP was $>260$ or $<70 \mathrm{~mm} \mathrm{Hg}$ or if the diastolic BP was $>150$ or $<40 \mathrm{~mm} \mathrm{Hg}$ and the pulse pressure was $>150$ or $<20 \mathrm{~mm} \mathrm{Hg} .{ }^{35}$ The subjects had recordings of good technical quality (at least $70 \%$ were valid readings).

In this population, the exploratory analysis showed that systolic BP was a better predictor of cardiovascular outcome than diastolic BP. Moreover, it has been reported that systolic BP is superior to diastolic BP in predicting the prognosis of older subjects. ${ }^{32,37}$ Thus, in the present study, we used systolic BP to define various parameters.

\section{Echocardiography}

Left atrial (LA) and left ventricular (LV) measurements and a calculation of the LV mass were made according to standardized methods. ${ }^{38}$ The LA diameter $(\mathrm{cm})$ was indexed by body surface area $\left(\mathrm{m}^{2}\right)$, and LA enlargement was defined as LA diameter/body surface area $\geqslant 2.4 \mathrm{~cm} \mathrm{~m}^{-2} .{ }^{27}$ The LV mass was indexed by height ${ }^{2.7}$, and LV hypertrophy was defined as LV mass/height ${ }^{2.7}>50 \mathrm{~g} \mathrm{~m}^{-2.7}$ for men and $>47 \mathrm{~g} \mathrm{~m}^{-2.7}$ for women. ${ }^{39}$ The LV EF was calculated using the Teichholz formula or the Simpson rule ${ }^{38}$ and defined as low when it was $<50 \%$.

\section{Follow-up}

Subjects were followed up in our hospital outpatient clinic or by their family doctors. The occurrence of cardiovascular events was recorded during follow-up visits or by a telephone interview of the patient followed by a clinical visit. Data were collected by the authors of this study. Those reviewing the end points were blinded to other patients' data. In the present report, we evaluated a combined end point that included fatal and non-fatal ischemic or hemorrhagic stroke, coronary events (that is, sudden death, fatal and non-fatal myocardial infarction and coronary revascularization), heart failure with reduced or preserved EF that required hospitalization and peripheral revascularization. The outcomes were defined as previously reported. ${ }^{28,30,31}$

\section{Statistical analysis}

Standard descriptive statistics were used. Groups were compared by using a one-way ANOVA followed by a multiple comparison test, a chi-square test or a Fisher's exact test with Bonferroni's correction, where appropriate. Event rates are expressed as the number of events per 100 patient-years on the basis of the ratio of the observed number of events to the total number of patient-years of exposure up to the terminating event or censor. A Cox regression analysis was used to evaluate the univariate and multivariate associations of factors with the outcomes. First, we evaluated the univariate associations between cardiovascular events and age, gender, body mass index, smoking status, previous events, diabetes, estimated glomerular filtration rate (eGFR), low-density lipoprotein cholesterol, LV hypertrophy, LA enlargement, asymptomatic LV systolic dysfunction at baseline, clinic BP, 24- $\mathrm{h}$ BP, daytime BP, nighttime BP, ambulatory BP groups (dippers with normal MS were the reference group, and dippers with a high MS, non-dippers, reverse dippers, extreme dippers with a normal MS and extreme dippers with a high MS were the compared groups), weighted systolic BP s.d., and antihypertensive, antiplatelet and statin therapy at baseline and atrial fibrillation during follow-up. Then, multiple regression analysis was performed, and we reported variables in the final model that were significantly $(P<0.05)$ associated with outcomes in the univariate analysis. The forced entry model was used. For ambulatory BP groups, the main topic of this study, we also calculated the population attributable risk percentage according to the following formula: ((incidence in the total populationincidence in the unexposed)/incidence in the total population) $\times 100$. Statistical significance was defined as $P<0.05$. The analyses were performed with the SPSS 21 software package (SPSS, Chicago, IL, USA). The graphs were made with GraphPad Prism 6 (GraphPad software, San Diego, CA, USA).

\section{RESULTS}

The characteristics and BP values of the study population are summarized in Table 1. Age, prevalence of LA enlargement, clinic BP, daytime BP, nighttime BP, 24-h BP, MS (by definition), percentage of dipping (by definition) and weighted systolic BP s.d. were significantly different among the groups. Age and prevalence of LA enlargement were the highest among the reverse dippers. Clinic and daytime systolic and diastolic BP were the highest among the dippers and the extreme dippers with a high MS. Nighttime systolic and diastolic BP were the highest among the reverse dippers and non-dippers. The 24-h systolic BP was the highest among the dippers with a high MS and the reverse dippers. The weighted systolic BP s.d. was the highest among the dippers with a high MS and the extreme dippers with a normal MS. The other variables were not significantly different among the groups.

The baseline data for antihypertensive therapy are reported in Table 2. The use of diuretics, beta-blockers, calcium antagonists, angiotensin-converting enzyme inhibitors and angiotensin receptor blockers and single and double therapy was not significantly different among the groups. The use of alpha-blockers and triple therapy was 
Table 1 Characteristics of study population

\begin{tabular}{|c|c|c|c|c|c|c|c|}
\hline Parameter & Reverse dippers & Non-dippers & Dippers (normal MS) & Dippers (high MS) & Extreme dippers (normal MS) & Extreme dippers (high MS) & $\mathrm{P}$ \\
\hline$n$ & 116 & 527 & 334 & 152 & 29 & 33 & \\
\hline Age, years & $70.8 \pm 6.4^{a}$ & $68.9 \pm 6.4$ & $67.8 \pm 6.3$ & $68.7 \pm 6.4$ & $66.9 \pm 5.7$ & $70.6 \pm 6.3$ & 0.0001 \\
\hline Men, $n(\%)$ & $59(51)$ & $227(43)$ & 132 (39) & $66(43)$ & $10(34)$ & $12(36)$ & 0.31 \\
\hline Body mass index, $\mathrm{kg} \mathrm{m}^{-2}$ & $27.5 \pm 3.7$ & $27.9 \pm 4.3$ & $27.5 \pm 4.1$ & $27.6 \pm 3.6$ & $28.5 \pm 4.5$ & $25.9 \pm 2.6$ & 0.09 \\
\hline Smokers, $n(\%)$ & $11(9)$ & $57(11)$ & $45(13)$ & $22(14)$ & $3(10)$ & $3(9)$ & 0.65 \\
\hline Previous events, $n(\%)$ & $12(10)$ & $52(10)$ & $20(6)$ & $13(9)$ & $4(14)$ & $3(9)$ & 0.38 \\
\hline Diabetes, $n(\%)$ & $21(18)$ & $66(12)$ & $35(10)$ & $19(12)$ & $2(7)$ & $5(15)$ & 0.34 \\
\hline eGFR, $\mathrm{ml} \mathrm{min} \mathrm{m}^{-1} 1.73 \mathrm{~m}^{-2}$ & $64.1 \pm 16$ & $64.5 \pm 15$ & $64.1 \pm 14$ & $63.3 \pm 16$ & $63 \pm 11$ & $64.3 \pm 13$ & 0.95 \\
\hline LDL cholesterol, $\mathrm{mg} \mathrm{dl}^{-1}$ & $123 \pm 33$ & $127 \pm 29$ & $130 \pm 30$ & $128 \pm 29$ & $128 \pm 34$ & $131 \pm 29$ & 0.37 \\
\hline LV hypertrophy, $n(\%)$ & $38(33)$ & $167(32)$ & $90(27)$ & $56(36)$ & $10(34)$ & $8(24)$ & 0.29 \\
\hline LA enlargement, $n(\%)$ & $46(40)^{a, b, c}$ & $123(23)^{a}$ & $50(15)$ & $33(22)$ & $6(21)$ & $5(15)$ & 0.0001 \\
\hline ALVSD, $n(\%)$ & $7(6)$ & $22(4)$ & $11(3)$ & $11(7)$ & $0(0)$ & $2(6)$ & 0.29 \\
\hline Clinic SBP, mm Hg & $147 \pm 16^{b}$ & $149 \pm 18^{b}$ & $148 \pm 18$ & $159 \pm 19^{a}$ & $151 \pm 16$ & $158 \pm 21$ & 0.0001 \\
\hline Clinic DBP, mm Hg & $85 \pm 11^{b}$ & $87 \pm 10^{\mathrm{b}}$ & $87 \pm 11$ & $91 \pm 9^{a}$ & $86 \pm 9$ & $92 \pm 80$ & 0.0001 \\
\hline Daytime SBP, mm Hg & $132.8 \pm 14^{\mathrm{b}}$ & $134.3 \pm 15^{b}$ & $134.3 \pm 14$ & $142.6 \pm 14^{a}$ & $135.8 \pm 13$ & $141.3 \pm 13$ & 0.0001 \\
\hline Daytime DBP, mm Hg & $76 \pm 7^{b}$ & $78 \pm 9^{b}$ & $78 \pm 9$ & $81 \pm 9$ & $77 \pm 7$ & $82 \pm 6$ & 0.0001 \\
\hline Nighttime SBP, mm Hg & $138 \pm 15^{a, b, c}$ & $127 \pm 14^{a, b}$ & $116 \pm 12$ & $122 \pm 12^{a}$ & $104 \pm 11^{a, b, c, d}$ & $108 \pm 12^{b, c, d}$ & 0.0001 \\
\hline Nighttime DBP, mm Hg & $75 \pm 8^{a, b, c}$ & $70 \pm 9^{a, b}$ & $65 \pm 8$ & $66 \pm 7$ & $57 \pm 6^{a, b, c, d}$ & $59 \pm 5^{\mathrm{a}, \mathrm{b}, \mathrm{c}, \mathrm{d}}$ & 0.0001 \\
\hline 24- h SBP, mm Hg & $134 \pm 14^{\mathrm{a}}$ & $132 \pm 14^{b}$ & $129 \pm 13$ & $136 \pm 13^{a}$ & $126 \pm 12^{b}$ & $131 \pm 12$ & 0.0001 \\
\hline 24- h DBP, mm Hg & $76 \pm 7$ & $75 \pm 9$ & $74 \pm 9$ & $76 \pm 8$ & $71 \pm 6$ & $75 \pm 6$ & 0.01 \\
\hline Morning surge SBP, mm Hg & $-2.5 \pm 8.2^{a, b, c}$ & $8.7 \pm 8.8^{a, b}$ & $13.7 \pm 5.7$ & $30.5 \pm 6.1^{a}$ & $17.5 \pm 4.2^{\mathrm{b}, \mathrm{c}, \mathrm{d}}$ & $33.6 \pm 9.6^{a, c, d, e}$ & 0.0001 \\
\hline Weighted SBP s.d., mm Hg & $13.7 \pm 3.5^{\mathrm{a}}$ & $12.7 \pm 3.1^{b}$ & $12.6 \pm 3.2$ & $14.4 \pm 3.2^{a}$ & $14.7 \pm 3.1^{\mathrm{a}}$ & $13.7 \pm 3.1$ & 0.0001 \\
\hline Dipping SBP, \% & $-4.3 \pm 3.7^{a, b, c}$ & $5.5 \pm 2.8^{a, b}$ & $13.6 \pm 2.5$ & $14.5 \pm 2.6$ & $23.3 \pm 3.1^{a, b, c, d}$ & $23.6 \pm 3.9^{a, b, c, d}$ & 0.0001 \\
\hline Atrial fibrillation, ${ }^{f} n(\%)$ & $10(9)$ & $45(8)$ & $21(6)$ & $18(12)$ & $1(3)$ & $0(0)$ & 0.14 \\
\hline
\end{tabular}

Abbreviations: ALVSD, asymptomatic left ventricular systolic dysfunction (EF <50\%); DBP, diastolic blood pressure; eGFR, estimated glomerular filtration rate; LA, left atrial; LDL, low-density lipoprotein; LV, left ventricular; MS, morning surge (high MS means SBP > $23 \mathrm{~mm} \mathrm{Hg}$ ); SBP, systolic blood pressure.

${ }^{a} P<0.05$ vs. dippers (normal MS).

${ }^{b} P<0.05$ vs. dippers (high MS)

${ }^{c} P<0.05$ vs. non-dippers.

$\mathrm{d} P<0.05$ vs. reverse dippers

$P<0.05$ vs. extreme dippers (normal MS)

${ }^{\mathrm{f}}$ Atrial fibrillation occurred during follow-up.

Table 2 Baseline antihypertensive therapy of study population

\begin{tabular}{|c|c|c|c|c|c|c|c|}
\hline Parameter & Reverse dippers & Non-dippers & Dippers (normal MS) & Dippers (high MS) & Extreme dippers (normal MS) & Extreme dippers (high MS) & $\mathrm{P}$ \\
\hline Diuretic, $n(\%)$ & $73(63)$ & $294(56)$ & $195(58)$ & $86(57)$ & $17(59)$ & $19(58)$ & 0.82 \\
\hline Beta-blocker, $n(\%)$ & $35(30)$ & $165(31)$ & $91(27)$ & $54(35)$ & $11(38)$ & $9(27)$ & 0.47 \\
\hline Calcium antagonist, $n(\%)$ & $48(11)$ & $190(36)$ & $97(29)$ & $44(29)$ & $9(31)$ & $13(39)$ & 0.08 \\
\hline ACE-I, $n(\%)$ & $68(59)$ & $262(50)$ & $162(48)$ & $85(56)$ & $13(45)$ & $11(33)$ & 0.08 \\
\hline ARB, $n(\%)$ & $24(21)$ & $130(25)$ & $92(27)$ & $30(20)$ & $9(31)$ & $10(30)$ & 0.34 \\
\hline Alpha-blocker, $n(\%)$ & $32(28)^{a, b, c}$ & $73(14)$ & $41(12)$ & $12(8)$ & $3(10)$ & $3(9)$ & 0.0001 \\
\hline Single therapy, $n(\%)$ & $20(17)$ & $134(25)$ & $92(27)$ & $33(22)$ & $4(14)$ & $8(24)$ & 0.18 \\
\hline Double therapy, $n(\%)$ & $48(41)$ & $236(45)$ & $159(48)$ & $82(54)$ & $18(62)$ & $19(58)$ & 0.09 \\
\hline Triple therapy, $n(\%)$ & $48(41)^{a, b}$ & $157(30)$ & $83(25)$ & $37(24)$ & $7(24)$ & $6(18)$ & 0.0001 \\
\hline
\end{tabular}

Abbreviations: ACE-I, angiotensin-converting enzyme inhibitor; ARB, angiotensin receptor blocker; MS, morning surge (high MS means SBP > 23 mm Hg); SBP, systolic blood pressure.

a $P<0.05$ vs. dippers (normal MS)

${ }^{b} P<0.05$ vs. dippers (high MS).

${ }^{c} P<0.05$ vs. non-dippers.

higher among the reverse dippers. At baseline, the use of aspirin and statin was not significantly different among the dippers with a normal MS, dippers with a high MS, non-dippers, reverse dippers, extreme dippers with a normal MS and extreme dippers with a high MS (for aspirin: $22 \%$ vs. $20 \%$ vs. $22 \%$ vs. $30 \%$ vs. $31 \%$ vs. $30 \%$, respectively, $P=0.23$; for statins: $11 \%$ vs. $11 \%$ vs. $10 \%$ vs. $16 \%$ vs. $21 \%$ vs. $24 \%$, respectively, $P=0.06$ ).

During the follow-up $(9.1 \pm 4.9$ years, range $0.4-20$ years $), 392$ cardiovascular events occurred. Specifically, there were 47 fatal and 92 non-fatal ischemic strokes, 5 fatal and 4 non-fatal hemorrhagic strokes, 35 fatal and 54 non-fatal myocardial infarctions, 31 coronary revascularizations, 123 heart failures (67 with a preserved EF and 56 with a reduced EF, 6 of which were preceded by a coronary event and 4 of which were preceded by a cerebrovascular event and were excluded from the total count) and 11 peripheral revascularizations. The event rate of the population as a whole was 3.63 per 100 patient-years. There were 65, 52, 189, 64, 6 and 16 events among the dippers with a normal MS, dippers with a high MS, non-dippers, reverse dippers, extreme dippers with a normal MS and extreme dippers with a high MS, respectively. 
The univariate analysis showed that age, diabetes, previous events, eGFR, LV hypertrophy, LA enlargement, asymptomatic LV systolic dysfunction at baseline, clinic systolic BP, 24-h systolic BP, daytime systolic BP, nighttime systolic BP, dippers with a high MS, non-dippers, reverse dippers and extreme dippers with a high MS were significantly associated with an increased cardiovascular risk (Table 3). There was no significant association between cardiovascular risk and other variables.

We performed two multivariate analyses. In the first model, we included age, diabetes, previous events, eGFR, LV hypertrophy, LA enlargement, asymptomatic LV systolic dysfunction at baseline and clinic systolic BP. In the second model, we replaced clinic BP with 24-h systolic BP and included circadian BP subgroups.

The results of the multivariate analyses are reported in Figure 1. After adjustment for the above-mentioned covariates, the risk of cardiovascular events was slightly associated with clinic BP (model 1), more strongly associated with 24-h BP and significantly higher among the dippers with a high MS, non-dippers, reverse dippers and extreme dippers with a high MS than among the dippers with a normal MS (model 2) (Figure 1).

When we assessed the effects of drug treatment at follow-up, the results were similar. When we analyzed the distribution of single components of the composite end point among circadian BP groups, we found that both stroke and coronary artery disease had a similar trend to that observed for all events; indeed, the highest incidence of both of these events was observed among the reverse dippers and extreme dippers with a high MS. The event rate of patients enrolled from 1992 to 2002 was similar to that of patients enrolled from 2003 to 2012. If enrollment year was included in the multivariate analysis, then the results were similar. When we separately analyzed patients with controlled $(n=418)$ or uncontrolled $(n=773)$ clinic systolic BP at baseline by using a cutoff of $140 \mathrm{~mm} \mathrm{Hg}$, the risk trend across circadian BP phenotypes was similar to that of the global population in both groups with a significantly higher risk among the non-dippers, reverse dippers and extreme dippers with a high MS;

Table 3 Risk of cardiovascular events in univariate analysis

\begin{tabular}{|c|c|c|}
\hline & $H R(95 \% \mathrm{Cl})$ & $\mathrm{P}$ \\
\hline Age (10 years) & $2.41(2.06-2.82)$ & 0.0001 \\
\hline Diabetes (yes vs. no) & $2.14(1.60-2.87)$ & 0.0001 \\
\hline Previous events (yes vs. no) & $1.86(1.29-2.68)$ & 0.001 \\
\hline eGFR (10 $\left.\mathrm{ml} \mathrm{min}^{-1} 1.73 \mathrm{~m}^{-2}\right)$ & $0.87(0.80-0.94)$ & 0.0001 \\
\hline LV hypertrophy (yes vs. no) & $2.14(1.75-2.61)$ & 0.0001 \\
\hline LA enlargement (yes vs. no) & $1.40(1.13-1.73)$ & 0.002 \\
\hline ALVSD (yes vs. no) & $2.45(1.71-3.51)$ & 0.0001 \\
\hline Clinic SBP (10 mm Hg) & $1.16(1.09-1.23)$ & 0.0001 \\
\hline 24-h SBP (10 mm Hg) & $1.38(1.30-1.46)$ & 0.0001 \\
\hline Daytime SBP (10 mm Hg) & $1.34(1.26-1.42)$ & 0.0001 \\
\hline Nighttime SBP (10 mm Hg) & $1.35(1.27-1.43)$ & 0.0001 \\
\hline \multicolumn{3}{|l|}{ Subgroups } \\
\hline Dippers (normal MS) & 1 & \\
\hline Dippers (high MS) & $2.14(1.48-3.08)$ & 0.0001 \\
\hline Non-dippers & $2.01(1.52-2.67)$ & 0.0001 \\
\hline Reverse dippers & $2.77(1.96-3.91)$ & 0.0001 \\
\hline Extreme dippers (normal MS) & $1.26(0.54-2.90)$ & 0.60 \\
\hline Extreme dippers (high MS) & $3.75(2.17-6.49)$ & 0.0001 \\
\hline
\end{tabular}

Abbreviations: ALVSD, asymptomatic left ventricular systolic dysfunction; $\mathrm{Cl}$, confidence interval; eGFR, estimated glomerular filtration rate; HR, hazard ratio; LA, left atrial; LV, left ventricular; MS, morning surge (high MS means $>23 \mathrm{~mm} \mathrm{Hg}$ ); SBP, systolic blood pressure. however, patients with controlled clinic BP had a globally lower event rate (2.85 per 100 patient-years) than those with uncontrolled clinic BP (4.13 per 100 patient-years).

The population attributable risk percentage was $0.6 \%$ among the dippers with a high MS, 7.1\% among non-dippers, $7.3 \%$ among reverse dippers and $1.4 \%$ among extreme dippers with a high MS.

\section{DISCUSSION}

This study shows that changes in circadian BP predict cardiovascular risk independently of $24-\mathrm{h}$ BP and other risk factors among elderly-treated hypertensive patients.

Our findings among reverse dippers are essentially in line with those of other studies evaluating the prognostic value of this specific pattern. $.^{9} 10,12,13,15-17,19-22$ Regarding non-dippers, our data are similar to those reported in some studies $6,7,10,12,14,16,18-20,22$ but different from those observed in other reports. ${ }^{9,13,15,17}$ It should be emphasized that we divided the dippers into those with a normal or high MS and non-dippers were compared with dippers with a normal MS who were at lower risk than those with a high MS. This aspect may explain the discrepancies between our data and some previous studies, ${ }^{9,13,15,17}$ as well as discrepancies among other studies that have observed differences ${ }^{6,7,10,12,14,16,18-20,22}$ or no differences ${ }^{9,13,15,17}$ between dippers and non-dippers regarding cardiovascular risk. Indeed, a different prevalence of dippers with a high MS within the dipper population may have affected the global risk of this group and the comparison with the non-dippers. Some previous studies evaluating cardiovascular outcome among all the extreme dippers compared with all dippers have reported increased risk, ${ }^{9,22}$ whereas others have reported no increased risk. ${ }^{10,15,19}$ Moreover, various studies have evaluated cardiovascular outcome among subjects with a high MS, independent of the circadian BP pattern, reporting conflicting results. ${ }^{8,19,22,25-27,29,32-34}$ To the best of our knowledge, this is the first study in the literature that compared cardiovascular risk among the dippers and extreme dippers with a high MS with that of dippers with a normal MS. In our previous studies, ${ }^{28,30,31}$ we have compared

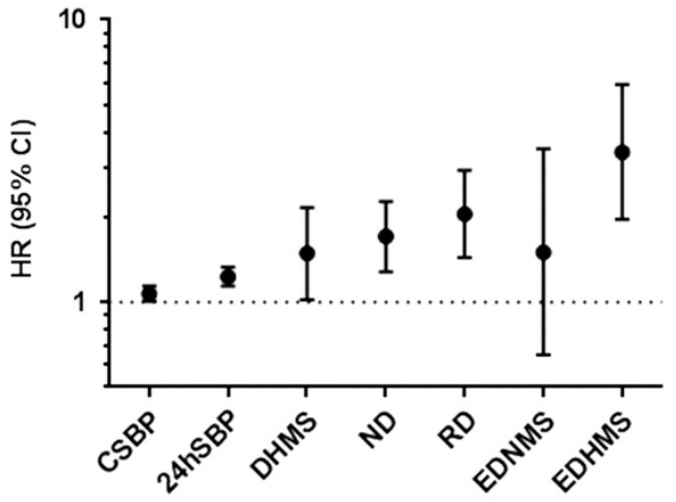

Figure 1 Risk of cardiovascular events by clinic and 24-h systolic blood pressure (CSBP and 24hSBP, respectively, per $10 \mathrm{~mm} \mathrm{Hg}$ increase) and among the dippers with a high morning surge (DHMS), non-dippers (ND), reverse dippers (RD), extreme dippers with a normal morning surge (EDNMS) and extreme dippers with a high morning surge (EDHMS) compared with dippers with a normal MS. The data are adjusted for age, diabetes, previous events, eGFR, LV hypertrophy, LA enlargement and asymptomatic LV systolic dysfunction at baseline. The hazard ratio (HR) and $95 \%$ confidence interval (Cl) values are $1.07(1.01-1.14)$ for CSBP, $1.23(1.14-1.33)$ for 24hSBP, 1.49 (1.02-2.16) among DHMS, 1.71 (1.28-2.27) among ND, 2.05 (1.44-2.93) among RD, 1.50 (0.65-3.49) among EDNMS and 3.40 (1.96-5.90) among EDHMS. 
the risk of stroke or coronary artery disease or heart failure among the dippers (including both dippers and extreme dippers) with a high MS and non-dippers (including both non-dippers and reverse dippers) with those of dippers with a normal MS (including both dippers and extreme dippers). In the present report, we found a higher risk among the dippers and extreme dippers with a high MS than among dippers with a normal MS, whereas the extreme dippers with a normal MS tended to have increased risk, but the association did not reach statistical significance. These findings highlight the prognostic relevance of the MS among both dippers and extreme dippers. They also suggest that the increased risk among the extreme dippers may be more associated with increased BP in the morning than with reduced $\mathrm{BP}$ at night; however, the non-significant increased risk observed among the extreme dippers with a normal MS deserves further evaluation because of the small number of events. A recent meta-analysis, ${ }^{40}$ which included our previously published study ${ }^{41}$ (1280 hypertensive patients of whom 57\% were treated, mean age 58 years, 104 events), has evaluated the prognostic effects of nocturnal BP fall among patients with hypertension. Reverse dippers and non-dippers are at increased cardiovascular risk compared with all dippers (with no distinction between those with a normal or high MS). ${ }^{40}$ Regarding extreme dippers (with no distinction between those with a normal or high MS), this BP pattern is associated with an increased cardiovascular risk among untreated populations but not among treated or mixed populations. ${ }^{40}$ Our present data on elderly-treated hypertensive patients are not consistent with this specific finding. However, the discrepancy may be explained by various aspects, including: (1) the younger age of the populations in our study and others (mean age of approximately 60 years), (2) the evaluation of treated/mixed populations, (3) the lack of distinction between dippers and extreme dippers with a normal or high MS (unavailable data for the meta-analysis), (4) the population characteristics and (5) other undetected factors.

Since the first descriptions of blunted nocturnal BP fall, ${ }^{6}$ extreme dipping ${ }^{42}$ and a high $\mathrm{MS},{ }^{25}$ there has been an attempt to explain their pathophysiological background and the mechanisms by which they are associated with increased cardiovascular risk. Various hypotheses have been formulated and discussed in previous reports. ${ }^{7,9,10,12-22,25-32,40,42-49}$ A blunted nocturnal BP fall has been shown to be associated with autonomic nervous system dysfunction, daytime orthostatic hypotension, neurohumoral alterations, elevated myocardial repolarization lability, increased salt sensitivity, sleeprelated breathing disorders, hyperaldosteronism, chronic low-grade inflammation, increased platelet activation, alterations in hemostasis, endothelial dysfunction, increased myocardial fibrosis and genetic variants in circadian genes. $7,9,10,12-22,40,42-47$ Extreme dipping has been reported to be associated with autonomic nervous system dysfunction, daytime orthostatic hypertension, neurohumoral alterations, a high MS and increased arterial stiffness. ${ }^{9,22,42-45}$ A high MS has been described to be associated with impaired baroreflexes, increased arterial stiffness, increased oxidative stress, chronic low-grade inflammation, increased platelet activation, alterations in hemostasis and coronary microvascular dysfunction. ${ }^{22,25-32,48,49}$ It should be emphasized that there is an overlap of different BP phenotypes at the extreme edges; indeed, extreme dipping is associated with a high MS and orthostatic hypertension, whereas reverse dipping is associated with an absent or inverse MS and orthostatic hypotension. ${ }^{49}$ These aspects highlight the complexity of the pathophysiological background and of the prognostic effects of different BP phenotypes and further suggest that some phenomena, such as non-dipping and MS, should be analyzed separately.
It is tempting to analyze which therapeutic strategy is the best at reducing $\mathrm{BP}$ and cardiovascular risk according to circadian $\mathrm{BP}$ changes. Indeed, it has been reported that antihypertensive therapy using clinic BP may be less effective in reducing risk for some circadian BP patterns, particularly among the reverse dippers and extreme dippers. ${ }^{50}$ It has been speculated that among the reverse dippers, maintaining BP control with clinic BP is insufficient during the night and that, among the extreme dippers, antihypertensive therapy may favor events by additionally reducing nocturnal BP or alternatively by partially controlling MS. ${ }^{50}$ Amlodipine administered at $0800 \mathrm{~h}$ has been shown to reduce BP during the night among patients with a high nighttime BP (non-dippers and reverse dippers) and to have no effect on nocturnal BP among those with a low nighttime BP (extreme dippers). ${ }^{51}$ Doxazosin administered at bedtime tends to increase nocturnal systolic BP among the extreme dippers, has a minimal effect among the dippers and substantially decreases nighttime BP among non-dippers and reverse dippers. ${ }^{52}$ Telmisartan has been reported to be superior to ramipril (both taken in the morning) in normalizing the circadian BP pattern to a dipper profile and reducing the MS of $\mathrm{BP} .{ }^{53}$ Nifedipine GITS administered at bedtime has been shown to decrease the prevalence of non-dipping and to reduce the MS of $\mathrm{BP} .{ }^{54}$ The aforementioned therapeutic approaches might be effective in reducing BP and risk among non-dippers, reverse dippers and extreme dippers with a high MS. It has been suggested that antihypertensive drugs administered at bedtime instead of the morning, for example, calcium channel blockers, angiotensin-converting enzyme inhibitors and angiotensinII receptor blockers, are more effective in reducing BP and risk, especially among patients with a high nighttime BP. ${ }^{55}$ This strategy may be mostly helpful among the non-dippers and reverse dippers. However, the best therapeutic approach to reduce cardiovascular risk, according to circadian BP profile, remains to be established. We found a significantly higher risk, though at a different rate, among the non-dippers, reverse dippers and extreme dippers with a high MS both among patients with controlled and uncontrolled clinic BP, thus suggesting that a therapeutic approach that targets the circadian BP pattern may be beneficial for both groups. Thus, drug therapy should be improved, though to a different extent, among the non-dippers, reverse dippers and extreme dippers with a high MS both when clinic BP is controlled and uncontrolled.

This study has some limitations. First, we studied only Caucasian subjects, and our results cannot be applied to other ethnic groups. Second, our data were obtained from elderly-treated hypertensive patients and cannot be extrapolated to younger and untreated subjects. Third, it remains unclear whether circadian BP changes entirely reflect an intrinsic characteristic of patients or uncontrolled BP because of the treatment features (dose or timing of drug therapy); these aspects, however, do not lessen our findings. Fourth, the lack of association of cardiovascular risk with treatment strategy does not indicate a lack of efficacy of therapy because all the subjects were treated with an antihypertensive therapy, most subjects received multiple therapies, and the patients were not randomized into antihypertensive, antiplatelet or statin therapy groups.

In conclusion, among elderly-treated hypertensive patients, circadian BP changes were independently associated with an increased cardiovascular risk. At the patient level, the highest risk was observed among the extreme dippers with a high MS, followed by the reverse dippers, non-dippers and dippers with a high MS. At the population level, the highest risk was observed among the reverse dippers, followed by the non-dippers, extreme dippers with a high MS and dippers with a high MS. Beyond using clinic BP, our findings suggest 
that using therapeutic strategies that improve nighttime BP reduction among reverse dippers and non-dippers and that reduce the MS of BP among patients with a high MS may possibly help to achieve strict 24-h BP control and help to better prevent cardiovascular events among elderly-treated hypertensive patients.

\section{CONFLICT OF INTEREST}

The authors declare no conflict of interest.

1 O'Brien E, Parati G, Stergiou G, Asmar R, Beilin L, Bilo G, Clement D, de la Sierra A de Leeuw P, Dolan E, Fagard R, Graves J, Head GA, Imai Y, Kario K, Lurb E, Mallion J-M, Mancia G, Mengden T, Myers M, Ogedegbe G, Ohkubo T, Omboni S, Palatini P, Redon J, Ruilope LM, Shennan A, Staessen JA, van Montfrans G, Verdecchia P, Waeber B, Wang J, Zanchetti A, Zhang Y. European Society of Hypertension Position Paper on ambulatory blood pressure monitoring. J Hypertens 2013; 31: 1731-1768.

2 Verdecchia P. Prognostic value of ambulatory blood pressure: current evidence and clinical implications. Hypertension 2000; 35: 844-851.

3 Pierdomenico SD, Lapenna D, Bucci A, Di Tommaso R, Di Mascio R, Manente BM, Caldarella MP, Neri M, Cuccurullo F, Mezzetti A. Cardiovascular outcome in treated hypertensive patients with responder, masked, false resistant, and true resistant hypertension. Am J Hypertens 2005; 18: 1422-1428.

4 Pierdomenico SD, Cuccurullo F. Prognostic value of white-coat and masked hypertension diagnosed by ambulatory monitoring in initially untreated subjects: an updated meta-analysis. Am J Hypertens 2011; 24: 52-58.

5 Roush GC, Fagard RH, Salles GF, Pierdomenico SD, Reboldi G, Verdecchia P, Eguchi K, Kario K, Hoshide S, Polonia J, de la Sierra A, Hermida RC, Dolan E, Zamalloa H, $\mathrm{ABC}-\mathrm{H}$ Investigators. Prognostic impact from clinic, daytime, and night-time systolic blood pressure in nine cohorts of 13,844 patients with hypertension. J Hypertens 2014; 32: 2332-2340.

6 O'Brien E, Sheridan J, O'Malley K. Dippers and non-dippers. Lancet 1988; 2: 397

7 Verdecchia P, Porcellati C, Schillaci G, Borgioni C, Ciucci A, Battistelli M, Guerrieri M, Gatteschi C, Zampi I, Santucci A, Santucci C, Reboldi G. Ambulatory blood pressure. An independent predictor of prognosis in essential hypertension. Hypertension 1994; 24: 793-801.

8 Staessen JA, Thijs L, Fagard R, O'Brien ET, Clement D, de Leeuw PW, Mancia G, Nachev C, Palatini P, Parati G, Tuomilehto J, Webster J. Predicting cardiovascular risk using conventional vs ambulatory blood pressure in older patients with systolic hypertension. Systolic Hypertension in Europe Trial Investigators. JAMA 1999; 282: 539-546.

9 Kario K, Pickering TG, Matsuo T, Hoshide S, Schwartz JE, Shimada K. Stroke prognosis and abnormal nocturnal blood pressure falls in older hypertensives. Hypertension 2001; 38: 852-857.

10 Ben-Dov IZ, Kark JD, Ben-Ishay D, Mekler J, Ben-Arie L, Bursztyn M. Predictors of all-cause mortality in clinical ambulatory monitoring: unique aspects of blood pressure during sleep. Hypertension 2007; 49: 1235-1241.

11 Fagard RH, Celis H, Thijs L, Staessen JA, Clement DL, De Buyzere ML, De Bacquer DA. Daytime and nighttime blood pressure as predictors of death and cause-specific cardiovascular events in hypertension. Hypertension 2008; 51: 55-61.

12 Fagard RH, Thijs L, Staessen JA, Clement DL, De Buyzere ML, De Bacquer DA. Prognostic significance of ambulatory blood pressure in hypertensive patients with history of cardiovascular disease. Blood Press Monit 2008; 13: 325-332.

13 Eguchi K, Pickering TG, Hoshide S, Ishikawa J, Ishikawa S, Schwartz JE, Shimada K, Kario K. Ambulatory blood pressure is a better marker than clinic blood pressure in predicting cardiovascular events in patients with/without type 2 diabetes. Am J Hypertens 2008; 21: 443-450.

14 Brotman DJ, Davidson MB, Boumitri M, Vidt DG. Impaired diurnal blood pressure variation and all-cause mortality. Am J Hypertens 2008; 21: 92-97.

15 Fagard RH, Thijs L, Staessen JA, Clement DL, De Buyzere ML, De Bacquer DA. Night-day blood pressure ratio and dipping pattern as predictors of death and cardiovascular events in hypertension. J Hum Hypertens 2009; 23: 645-653.

16 Muxfeldt ES, Cardoso CR, Salles GF. Prognostic value of nocturnal blood pressure reduction in resistant hypertension. Arch Intern Med 2009; 169: 874-880.

17 Bastos JM, Bertoquini S, Polónia J. Prognostic value of subdivisions of nighttime blood pressure fall in hypertensives followed up for 8.2 years. Does nondipping classification need to be redefined? J Clin Hypertens (Greenwich) 2010; 12: 508-515.

18 Hansen TW, Li Y, Boggia J, Thijs L, Richart T, Staessen JA. Predictive role of the nighttime blood pressure. Hypertension 2011; 57: 3-10.

19 Verdecchia P, Angeli F, Mazzotta G, Garofoli M, Ramundo E, Gentile G, Ambrosio G, Reboldi G. Day-night dip and early-morning surge in blood pressure in hypertension: prognostic implications. Hypertension 2012; 60: 34-42.

20 Ohkubo T, Imai Y, Tsuji I, Nagai K, Watanabe N, Minami N, Kato J, Kikuchi N, Nishiyama A, Aihara A, Sekino M, Satoh H, Hisamichi S. Relation between nocturnal decline in blood pressure and mortality. The Ohasama Study. Am J Hypertens 1997; 10: 1201-1207.

21 Ingelsson E, Björklund-Bodegård K, Lind L, Arnlöv J, Sundström J. Diurnal blood pressure pattern and risk of congestive heart failure. JAMA 2006; 295: 2859-2866.
22 Metoki H, Ohkubo T, Kikuya M, Asayama K, Obara T, Hashimoto J, Totsune K, Hoshi H, Satoh H, Imai Y. Prognostic significance for stroke of a morning pressor surge and a nocturnal blood pressure decline: the Ohasama study. Hypertension 2006; 47: $149-154$.

23 Muller JE, Tofler GH, Stone PH. Circadian variation and triggers of onset of acute cardiovascular disease. Circulation 1989; 79: 733-743.

24 Elliott WJ. Circadian variation in the timing of stroke onset: a meta-analysis. Stroke 1998; 29: 992-996.

25 Kario K, Pickering TG, Umeda Y, Hoshide S, Hoshide Y, Morinari M, Murata M, Kuroda T, Schwartz JE, Shimada K. Morning surge in blood pressure as a predictor of silent and clinical cerebrovascular disease in elderly hypertensives: a prospective study. Circulation 2003; 107: 1401-1406.

26 Gosse P, Lasserre R, Minifié C, Lemetayer P, Clementy J. Blood pressure surge on rising. J Hypertens 2004; 22: 1113-1118.

27 Turak O, Afsar B, Ozcan F, Canpolat U, Grbovic E, Mendi MA, Oksuz F, Siriopol D, Covic A, Caliskan M, McFann K, Johnson RJ, Kanbay M. Relationship between elevated morning blood pressure surge, uric acid, and cardiovascular outcomes in hypertensive patients. J Clin Hypertens (Greenwich) 2014; 16: 530-535.

28 Pierdomenico SD, Pierdomenico AM, Cuccurullo F. Morning blood pressure surge, dipping, and risk of ischemic stroke in elderly patients treated for hypertension. Am J Hypertens 2014; 27: 564-570.

29 Amodeo C, Guimarães GG, Picotti JC, dos Santos CC, Bezzerra Fonseca KD, Matins RF, Cordeiro AC, Mores Rego Sousa AG. Morning blood pressure surge is associated with death in hypertensive patients. Blood Press Monit 2014; 19: 199-202.

30 Pierdomenico SD, Pierdomenico AM, Di Tommaso R, Coccina F, Di Carlo S, Porreca E, Cuccurullo F. Morning Blood Pressure Surge, Dipping, and Risk of Coronary Events in Elderly Treated Hypertensive Patients. Am J Hypertens 2016; 29: 39-45.

31 Pierdomenico SD, Pierdomenico AM, Coccina F, Lapenna D, Porreca E. Ambulatory blood pressure parameters and heart failure with reduced or preserved ejection fraction in elderly treated hypertensive patients. Am J Hypertens (e-pub ahead of print 18 March 2016).

32 Li Y, Thijs L, Hansen TW, Kikuya M, Boggia J, Richart T, Metoki H, Ohkubo T, Torp-Pedersen C, Kuznetsova T, Stolarz-Skrzypek K, Tikhonoff V, Malyutina S, Casiglia E, Nikitin Y, Sandoya E, Kawecka-Jaszcz K, Ibsen H, Imai Y, Wang J, Staessen JA. International Database on Ambulatory Blood Pressure Monitoring in Relation to Cardiovascular Outcomes Investigators. Prognostic value of the morning blood pressure surge in 5645 subjects from 8 populations. Hypertension 2010; 55: 1040-1048.

33 Israel S, Israel A, Ben-Dov IZ, Bursztyn M. The morning blood pressure surge and all-cause mortality in patients referred for ambulatory blood pressure monitoring. Am J Hypertens 2011; 24: 796-801.

34 Bombelli M, Fodri D, Toso E, Macchiarulo M, Cairo M, Facchetti R, Dell'Oro R, Grassi G, Mancia G. Relationship among morning blood pressure surge, 24- hour blood pressure variability, and cardiovascular outcomes in a white population. Hypertension 2014; 64: 943-950.

35 Pierdomenico SD, Lapenna D, Guglielmi MD, Antidormi T, Schiavone C, Cuccurullo F, Mezzetti A. Target organ status and serum lipids in patients with white coat hypertension. Hypertension 1995; 26: 801-807.

36 Bilo G, Giglio A, Styczkiewicz K, Caldara G, Maronati A, Kawecka-Jaszcz K, Mancia G, Parati G. A new method for assessing $24 \mathrm{~h}$ blood pressure variability after excluding the contribution of nocturnal blood pressure fall. J Hypertens 2007; 25: 2058-2066.

37 Franklin SS, Larson MG, Khan SA, Wong ND, Leip EP, Kannel WB, Levy D. Does the relation of blood pressure to coronary heart disease risk change with aging? The Framingham Heart Study. Circulation 2001; 103: 1245-1249.

38 Lang RM, Bierig M, Devereux RB, Flachskampf FA, Foster E, Pellikka PA, Picard MH, Roman MJ, Seward J, Shanewise JS, Solomon SD, Spencer KT, Sutton MS, Stewart WJ, Chamber Quantification Writing Group, American Society of Echocardiography's Guidelines and Standards Committee, European Association of Echocardiography. Recommendations for chamber quantification: a report from the American Society of Echocardiography's Guidelines and Standards Committee and the Chamber Quantification Writing Group, developed in conjunction with the European Association of Echocardiography, a branch of the European Society of Cardiology. J Am Soc Echocardiogr 2005; 18: 1440-1463.

39 de Simone G, Devereux RB, Daniels SR, Koren MJ, Meyer RA, Laragh JH. Effect of growth on variability of left ventricular mass: assessment of allometric signals in adults and children and their capacity to predict cardiovascular risk. J Am Coll Cardiol 1995; 25: 1056-1062.

40 Salles GF, Reboldi G, Fagard RH, Cardoso CRL, Pierdomenico SD, Verdecchia P, Eguchi K, Kario K, Hoshide S, Polonia J, de la Sierra A, Hermida RC, Dolan E, O'Brien E, Roush GC, The ABC-H Investigators. Prognostic impact of the nocturnal blood pressure fall in hypertensive patients: the ambulatory blood pressure collaboration in patients with hypertension (ABC-H) meta-analysis. Hypertension 2016; 67: 693-700.

41 Pierdomenico SD, Di Nicola M, Esposito AL, Di Mascio R, Ballone E, Lapenna D, Cuccurullo $\mathrm{F}$. Prognostic value of different indices of blood pressure variability in hypertensive patients. Am J Hypertens 2009; 22: 842-847.

42 Kario K, Matsuo T, Kobayashi H, Imiya M, Matsuo M, Shimada K. Nocturnal fall of blood pressure and silent cerebrovascular damage in elderly hypertensive patients. Advanced silent cerebrovascular damage in extreme dippers. Hypertension 1996; 27: $130-135$. 
43 Kario K, Motai K, Mitsuhashi T, Suzuki T, Nakagawa Y, Ikeda U, Matsuo T, Nakayama T, Shimada K. Autonomic nervous system dysfunction in elderly hypertensive patients with abnormal diurnal blood pressure variation: relation to silent cerebrovascular disease. Hypertension 1997; 30: 1504-1510.

44 Kario K, Eguchi K, Nakagawa Y, Motai K, Shimada K. Relationship between extreme dippers and orthostatic hypertension in elderly hypertensive patients. Hypertension 1998; 31: 77-82.

45 Kario K, Mitsuhashi T, Shimada K. Neurohumoral characteristics of older hypertensive patients with abnormal nocturnal blood pressure dipping. Am J Hypertens 2002; 15 531-537.

46 Fagard RH. Dipping pattern of nocturnal blood pressure in patients with hypertension. Expert Rev Cardiovasc Ther 2009; 7: 599-605.

47 Leu HB, Chung CM, Lin SJ, Chiang KM, Yang HC, Ho HY, Ting CT, Lin TH, Sheu SH, Tsai WC, Chen JH, Yin WH, Chiu TY, Chen Cl, Fann CS, Chen YT, Pan WH, Chen JW. Association of circadian genes with diurnal blood pressure changes and non-dipper essential hypertension: a genetic association with young-onset hypertension. Hypertens Res 2015; 38: 155-162.

48 Kario K. Morning surge in blood pressure and cardiovascular risk: evidence and perspectives. Hypertension 2010; 56: 765-773.

49 Kario K. Prognosis in relation to blood pressure variability: pro side of the argument. Hypertension 2015; 65: 1163-1169.
50 Hoshide Y, Kario K, Schwartz JE, Hoshide S, Pickering TG, Shimada K. Incomplete benefit of antihypertensive therapy on stroke reduction in older hypertensives with abnormal nocturnal blood pressure dipping (extreme-dippers and reverse-dippers). Am J Hypertens 2002; 15 (10 Pt 1): 844-850.

51 Kario K, Shimada K. Differential effects of amlodipine on ambulatory blood pressure in elderly hypertensive patients with different nocturnal reductions in blood pressure. Am J Hypertens 1997; 10: 261-268.

52 Kario K, Shimada K. Risers and extreme-dippers of nocturnal blood pressure in hypertension: antihypertensive strategy for nocturnal blood pressure. Clin Exp Hypertens 2004; 26: 177-189.

53 Gosse P, Schumacher H. Effect of telmisartan vs. ramipril on 'dipping' status and blood pressure variability: pooled analysis of the PRISMA studies. Hypertens Res 2014; 37: 151-157.

54 Hermida RC, Ayala DE, Mojón A, Alonso I, Fernández JR. Reduction of morning blood pressure surge after treatment with nifedipine GITS at bedtime, but not upon awakening, in essential hypertension. Blood Press Monit 2009; 14: 152-159.

55 Hermida RC, Ayala DE, Smolensky MH, Fernández JR, Mojón A, Portaluppi F. Chronotherapy with conventional blood pressure medications improves management of hypertension and reduces cardiovascular and stroke risks. Hypertens Res 2016; 39 277-292. 